Article

\title{
Cell Stress Reduction by a Novel Perfusion-Culture System Using Commercial Culture Dish
}

\author{
Reiko Yasuda ${ }^{1,2}$, Shungo Adachi ${ }^{3}{ }^{\circledR}$, Atsuhito Okonogi ${ }^{2}$, Yohei Anzai ${ }^{2}$, Tadataka Kamiyama ${ }^{2}$, \\ Keiji Katano ${ }^{2}$, Nobuhiko Hoshi ${ }^{1}$, Katsuo Mogi ${ }^{3, *}$ and Tohru Natsume ${ }^{3, *}$ \\ 1 Laboratory of Animal Molecular Morphology, Department of Animal Science, Graduate School of Agricultural \\ Science, Kobe University, Kobe 657-8501, Japan; r.yasuda@icomes.co.jp (R.Y.); nobhoshi@kobe-u.ac.jp (N.H.) \\ 2 Icomes Lab Co., Ltd., Iwate 020-0857, Japan; a.okonogi@icomes.co.jp (A.O.); y.anzai@icomes.co.jp (Y.A.); \\ t.kamiyama@icomes.co.jp (T.K.); katano@icomes.co.jp (K.K.) \\ 3 Molecular Profiling Research Center for Drug Discovery, National Institute of Advanced Industrial Science \\ and Technology, Tokyo 135-0064, Japan; s.adachi@aist.go.jp \\ * $\quad$ Correspondence: mogi.k@aist.go.jp (K.M.); t-natsume@aist.go.jp (T.N.); Tel.: +81-3-3599-8251 (K.M.); \\ $+81-3-3599-8100$ (T.N.)
}

Received: 6 November 2019; Accepted: 18 December 2019; Published: 20 December 2019

\begin{abstract}
Two typical medium replacement methods are employed to culture cells, that is, traditional manual medium change, wherein complete medium changes are performed at short-term intervals; and perfusion, wherein continuous culture media delivery and discharge are performed. The former is associated with the issue of readily accumulation of metabolic waste products, which is resolved by the latter. However, the latter requires a specific chamber for cell culturing such as a microfluidic channel for flow stability. Generally, bio-medical researchers require a culturing process that utilizes commercial culture dishes to test their conventional manipulation know-how, experience, and protocols. Thus, we constructed a perfusion-culture system using commercial $35 \mathrm{~mm}$ culture dishes. To use commercial culture dishes, it is necessary to maintain the culturing conditions including a constant volume and uniform flow rate, and we invented a novel adapter, culture dish adapter (CD-Adapter), attached to a commercial culture dish. The CD-Adapter is fixed with a holding jig to keep the medium volume constant, so it can maintain a uniform flow in the culture dish. Additionally, we demonstrate the applicability of the system by applying it to a perfusion culture of $293 \mathrm{~T}$ cells, revealing a 50\% reduction in DDIT3, a cellular stress marker, compared with that in conventional manual medium change.
\end{abstract}

Keywords: manual medium change; perfusion culture; cellular stress; cell culture system; microfluidic device

\section{Introduction}

Technologies for cultivating animal cells in vitro (in an artificial environment) are called cell culture techniques [1]. One of the major advantages of cultivating cells in vitro is that one can control the cell culture environment parameters in the physical environment, such as temperature, $\mathrm{pH}$, and shear stress, as well as those in the physiological environment, including hormone or metabolic waste product concentrations [2-6]. In vitro cell culturing also allows researchers to analyze the phenomena of life in an experimental system, which is easier than analyzing cells in vivo [7]. To repeatedly obtain stable experimental results by fully exploiting these advantages, it is necessary to apply methods that effectively culture homogeneous cells with good reproducibility (high-quality cells) [8].

Ordinarily, it is necessary to regularly replace the culture medium when cultivating cells. Currently, this method of cell culture, known as manual medium change, is widely used [9]. Manual medium change 
is a method that involves completely removing the culture medium and replacing it with new media. However, in manual medium change, it is easy for waste products to accumulate and for nutrients to be replenished, which may cause cell stress owing to conditions such as acidosis, which lowers the quality of the cells [10]. Additionally, the culture medium is replaced manually within a biosafety cabinet outside the incubator, which exposes the culture to the external environment. In this way, manual medium change involves large changes in the conditions of the culture environment that may differ greatly from those in the in vivo environment in a way that is detrimental to the cells (Figure 1).

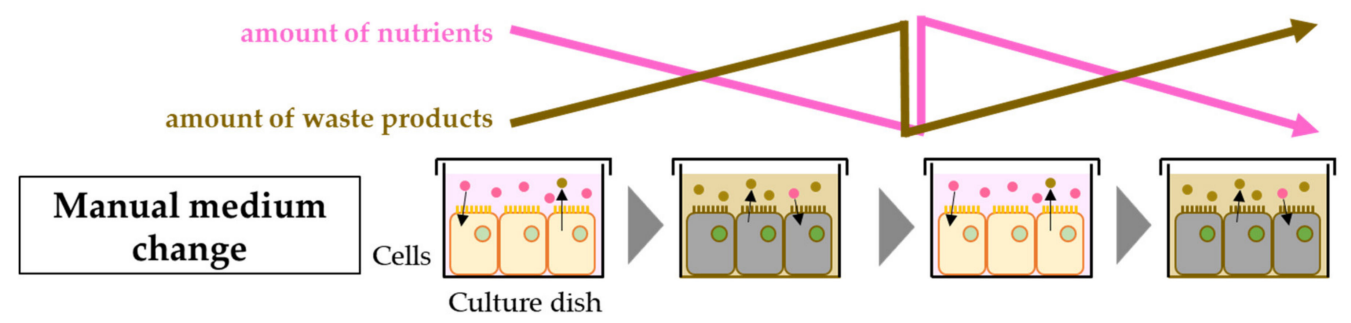

\section{Perfusion culture}

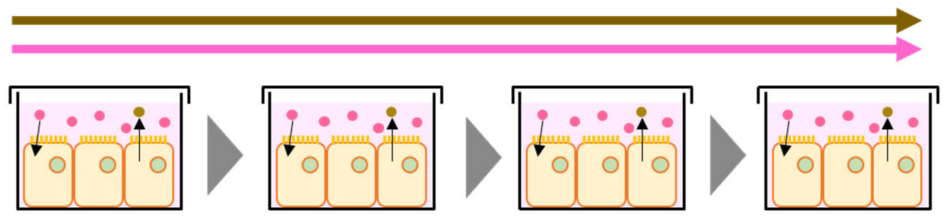

Figure 1. Cell culture environments in manual medium change and perfusion culture. In manual medium change, it is easy for waste products to accumulate and nutrients to be replenished, which may cause undue stress to the cells owing to conditions such as acidosis, thereby lowering the quality of the cells. Perfusion culture that constantly supplies and discharges the medium readily resolves these issues.

On the other hand, perfusion culture, which involves continuous delivery and discharge of culture medium, easily overcomes the issues of unstable medium conditions due to the manual medium change. The most important components of the perfusion culture are specific enclosed containers for cell culture, such as microfluidic devices or flow chambers. Although the superiority has not been sufficiently assessed, perfusion culture is expected to cause lower stress levels in cells compared with manual medium change. Perfusion culture can be performed easily by setting-up those containers and the related apparatus in an incubator [11,12]. However, in the laboratory, for retaining cells and allowing them to proliferate, commercial disposable culture dishes are widely used as containers [13]. The size of the culture dish varies for each manufacturing company. For example, the inner diameter of $35 \mathrm{~mm}$ culture dish from IWAKI is $35 \mathrm{~mm}$, but that from Nunc is $33.6 \mathrm{~mm}$. Researchers hesitate in changing the cell culturing condition formed in each dish because they have a knowledge of the cell culturing process for each culture dish. Using a different culturing container requires additional basic study for bridging the gap between conventional and new protocols. Hence, a new perfusion culturing system applicable to commercially available culture dishes is required to utilize existing resources and knowledge. To this end, several perfusion-culture systems employing commercial culture dishes have been reported in the literature, but these systems still serve as research platforms specialized for specific applications [14,15]. RC-37 Series (Warner Instruments) and Hiper-Go (LUMITOS AG) are commercially available; however, it is not easy to change the configuration flexibly to adjust to various experimental conditions.

In this study, we developed a versatile system for perfusion culture that can be easily equipped with various commercial $35 \mathrm{~mm}$ culture dishes. To test the system, we evaluated the "holding jig" component, which is one of the key components of the system, to prevent medium leakage and culturing container deformation. Additionally, we applied the system to culture 293T cells to verify the 
improvement of stress levels in cells. We compared the expression levels of DDIT3, which is known as the cellular stress marker gene, with those in cells cultured under manual medium change conditions.

\section{Materials and Methods}

\subsection{Study Design}

We constructed a perfusion culture system that was compatible with commercial culture dishes based on a tube pump (Icomes Lab, Iwate, Japan) (Figure 2A). Tube pumps are well suited for perfusion cultures because they are not limited by reservoir size or the amount of time for which they can operate; moreover, they have a low minimum flow rate and low dead volume, and do not require any preprocessing of the culture medium. An adapter named as culture dish adapter (CD-Adapter) was mounted on the culture dish (cat. no. 3000-35; AGC Techno Glass Co., Ltd., Shizuoka, Japan) [16]. The CD-Adapter has a function of sealing the culture dish to ensure a constant medium volume in the dish. Culture medium in a reservoir can be constantly injected into the closed dish using the pump. The tubes connecting the reservoir, the pump, the CD-Adapter, and a waste were polytetrafluoroethylene (PTFE) tubes with an inner diameter of $0.5 \mathrm{~mm}$ (TUF-100; Chukoh Chemical Industries, Ltd., Tokyo, Japan). Between the pump and the CD-Adapter, a filter with a pore size of $0.2 \mu \mathrm{m}$ (SLLGH04NL; Merck Millipore, MA, USA) was connected for bubble removal. A $50 \mathrm{~mL}$ tube (cat. no. 52070; Corning, New York, NY, USA) with a silicone rubber stopper (cat. no. 1-7650-02; AS ONE Corp., Osaka, Japan) was used as a reservoir. The flow rate was controlled by a software on a laptop PC, which was connected to the tube pump.

The CD-Adapter was fabricated by molding of polydimethylsiloxane (PDMS, CAT-106F, Shin-Etsu Chemical), which was cured at room temperature $\left(25^{\circ} \mathrm{C}\right)$. The mold master was made of epoxy resin (STYCAST 126J PTA, Henkel, Dusseldorf, Germany), as shown in Figure 2C, the fabrication method of which was based on a study by Yasuda et al. [16]. The center of the CD-Adapter attached to the culture medium is a $1 \mathrm{~mm}$ thick membrane, as shown in Figure 2B, to improve the gas permeability of PDMS to maintain the cell condition.

The medium volume in the dish can be changed by adjusting its height with a spacer, as shown in Figure 2B; in our case, a $2 \mathrm{~mm}$ spacer was used to design a $2 \mathrm{~mm}$ high container.

On the other hand, although the dimensions of commercial dishes differ slightly depending on the manufacturer, we could apply the CD-Adapter without leakage, to all commercial dishes using a holding jig (Figure 2D). The jig made of acrylic is attached to the center membrane of the CD-Adapter (Figure 2B).

\subsection{Study of Culture Media Pumping Methods and Evaluation of CD-Adapter Deflection}

In this study, a holding jig was attached not only for the tight sealing, but also for maintaining the shape of the CD-Adapter. Actually, an unintended deformation of the center membrane caused by a slight change in pressure could disrupt the stable culture environment, as a uniform flow cannot be maintained in the culture dish. Therefore, we devised a technique to prevent the deformation using an acrylic holing jig. The holding jig was placed on the center membrane, as shown in Figure 3 , functioning to restrain the membrane. To demonstrate the efficacy of the holding jig, the difference between lines $a$ and $b$ of Figure 3 was measured as an indicator of deformation.

The setup used for evaluation was composed of a culture dish, a $2 \mathrm{~mm}$ spacer, and a CD-Adapter, the outlet of which is closed and the inlet is attached to a syringe. The differences between a and $b$ of Figure 3 were measured at each pressure applied by a $10 \mathrm{~mL}$ syringe. Under experimental conditions at a given temperature of $25^{\circ} \mathrm{C}$, the difference in pressure was estimated using Equation (1) from Boyle's law:

$$
\Delta P=\frac{k}{\Delta V},
$$

where $\Delta P$ is the pressure difference in the culture space, $\Delta V$ is the difference in the volume, and $k$ is a constant. 
A
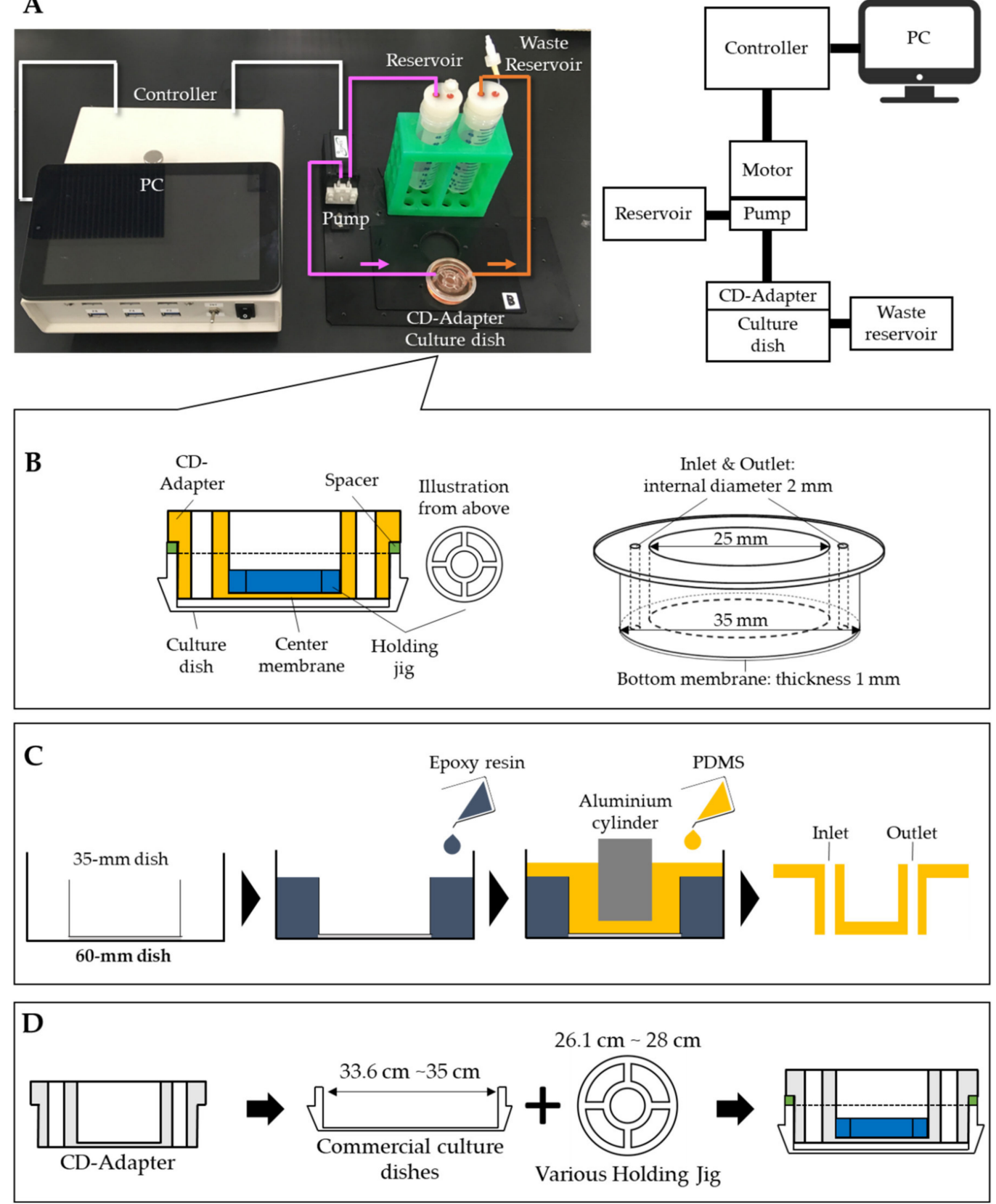

Figure 2. System overview and CD-Adapter. (A) Overview of the perfusion culture system developed in this study. (B) Dimension of the CD-Adapter. The size of the culturing area is adjusted by a spacer (green colored). The tight sealing is realized by the holding jig (blue colored), which presses the surface of the CD-Adapter against the dish wall. Deformation of the center membrane of the CD-Adapter is also prevented by the holding jig. (C) Fabrication process of the CD-Adapter. CD-Adapter is made by polydimethylsiloxane (PDMS) molding. The mold master is made of epoxy resin. The center membrane was formed by aluminum cylinder (gray colored). (D) Adaptation of the CD-Adapter to various $35 \mathrm{~mm}$ culture dishes. By adjusting the holding jig, the PDMS CD-Adapter is expanded and contracted, and the CD-Adapter can adapt to $35 \mathrm{~mm}$ culture dishes of different sizes. 


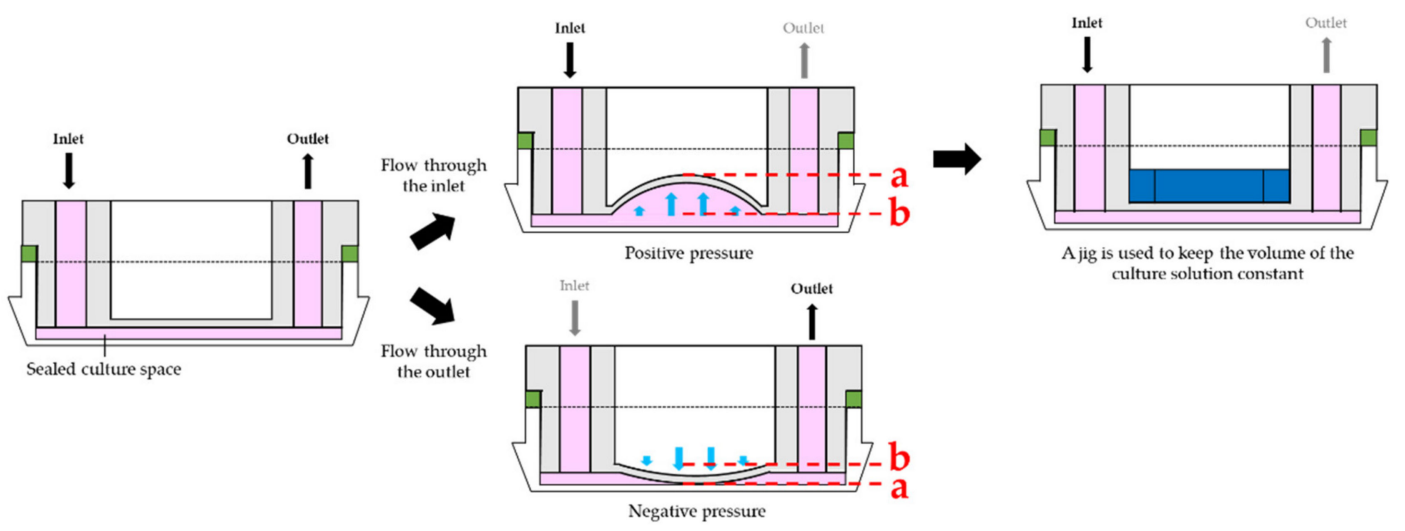

Figure 3. Deflection of the center membrane owing to liquid pumping. When pumping liquid into the culture dish, the center membrane swells, preventing the culture medium from being maintained at a constant volume in the dish. Additionally, discharging the culture medium causes the center membrane to become concave and may cause the membrane to touch the bottom of the culture dish. Thus, a holding jig was inserted into the CD-Adapter while pumping liquid into the culture dish to prevent physical swelling of the center membrane.

\subsection{Evaluation of The Stress Marker DDIT3 Expression in Perfusion Culture and Manual Medium Change}

To demonstrate that stable cell culture is possible using the developed perfusion-culture system, we evaluated cell stress by measuring the expression of the cellular stress marker DDIT3, which is also known as CHOP (C/EBP homologous protein). CHOP is related to endoplasmic reticulum stress [17], and is involved in promoting apoptosis, ultimately causing cell death [18].

As a preparation for the evaluation, human embryonic kidney epithelial cells (293T cells), a general culture cell line, were seeded at a density of $2 \times 10^{5}$ cells $/ \mathrm{mL}$ in $35 \mathrm{~mm}$ culture plates and cultured for one day at $37{ }^{\circ} \mathrm{C}$ in a $5 \% \mathrm{CO}_{2}$ environment. Thereafter, $2 \mathrm{~mL}$ Dulbecco's modified Eagle's medium, supplemented with $10 \%$ fetal bovine serum and used as the culture medium, was added to the culture dish. This evaluation experiment was conducted under three culture conditions, including perfusion culture, manual medium change (replacing the culture medium once per day), and culturing without replacing the culture medium as a negative control. To standardize the shape of the culture container, CD-Adapters were applied to all dishes. The flow rate for perfusion culture was $1.4 \mu \mathrm{L} / \mathrm{min}$, as this is the flow rate required for exchanging $2 \mathrm{~mL}$ of culture medium in one day. After culturing the cells for one and three days under each condition, the cells were collected by trypsinization using trypsin-ethylenediaminetetraacetic acid (EDTA), and the cells cultured for two days were counted. After cell collection, total RNA was extracted by RNeasy Plus Mini Kit (74134, QIAGEN, Hilden, Germany) and cDNA was synthesized for $120 \mathrm{~min}$ at $37^{\circ} \mathrm{C}$, and the enzyme was inactivated at $85^{\circ} \mathrm{C}$ for $5 \mathrm{~s}$ by High-Capacity cDNA Reverse Transcription Kit (4368814, Thermo Fisher scientific, Waltham, MA, USA) according to the protocol. DDIT3 expression was measured using reverse transcription polymerase chain reaction (qRT-PCR; $n=3$ ). According to the protocol, for qRT-PCR, denaturing was performed at $95^{\circ} \mathrm{C}$ for $23 \mathrm{~s}$, as well as annealing and extending at $60{ }^{\circ} \mathrm{C}$ for $30 \mathrm{~s}$, and this was performed for 40 cycles. The primer sequences used for qRT-PCR are presented in Table 1. $\beta$-Actin was used as an internal standard. Cell deviation and qRT-PCR results of DDIT3 were calculated as standard deviation.

Table 1. Primers used for reverse transcription polymerase chain reaction (qRT-PCR).

\begin{tabular}{ccc}
\hline Gene & \multicolumn{1}{c}{ Primer-Forward } & Primer-Reverse \\
\hline$\beta$-Actin & $5^{\prime}$-TGGATCAGCAAGCAGGAGTATG-3' & $5^{\prime}$-GCATTTGCGGTGGACGAT-3' \\
\hline DDIT3 & $5^{\prime}$-TCAGAGCTGGAACCTGAGGA-3' & $5^{\prime}$-CTGCCATCTCTGCAGTTGGA-3' \\
\hline
\end{tabular}




\section{Results and Discussion}

\subsection{Analysis of Culture Medium Pumping Methods and Evaluation of CD-Adapter Deflection}

Figure 4 shows the result of relationships between pressure on the CD-Adapter and displacement of the center membrane. The position of the center membrane at atmospheric pressure $(100 \mathrm{kPa})$ was defined as 0 . The average value of the three-measurement data was plotted as the displacement. As shown in Figure 4, precipitous deformation of the membrane was confirmed between $79 \mathrm{kPa}$ and $120 \mathrm{kPa}$. This result means that the container volume can be easily changed by the feeding pressure of the culture medium. In contrast, precipitous deformation by positive pressure was prevented by applying the holding jig, as shown in dotted line " $a$ " of Figure 4.

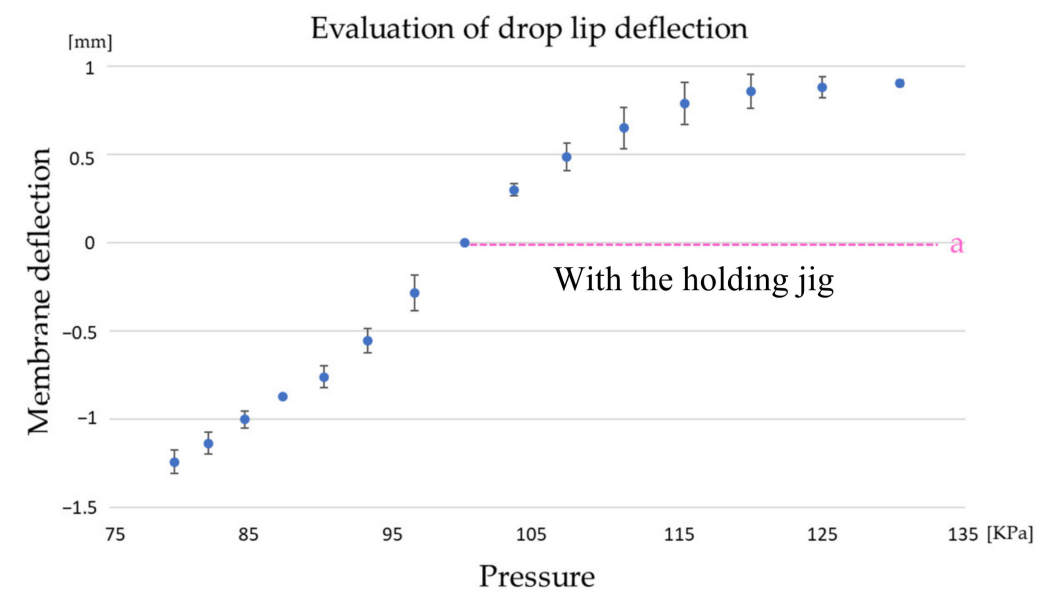

Figure 4. Evaluation result of the pressure and the deflection of the center membrane. The deflection is defined as 0 at atmospheric pressure $(100 \mathrm{kPa})$. Dotted line " $a$ " is the deflection of the membrane with the holding jig.

\subsection{Evaluation of the Expression of the Stress Marker DDIT3 in Perfusion Culture and Manual Medium Change}

The expression of the stress marker DDIT3 by $293 \mathrm{~T}$ cells cultured under perfusion, manual medium change, and no medium replacement conditions is presented in Figure 5B. The results reveal that, in perfusion culture, the relative expression levels were 1.00 on the inlet side and 1.23 on the outlet side. Similarly, the relative expression levels for manual medium change were 1.94 and 2.40, respectively, and those for no culture replacement conditions were 9.20 and 8.94, respectively. Compared with the results of the perfusion condition, the no culture replacement condition and the manual medium change condition showed an approximate nine-fold and two-fold increase in DDIT3 expression, respectively, indicating that perfusion culture reduced the amount of stress on cells compared with conventional manual medium change. Furthermore, we demonstrated that, under all the examined conditions, DDIT3 expression was almost equal in cells growing on the inlet and outlet sides. In perfusion culture using the system described in this paper, we showed that the cell culture environment was uniform throughout the culture dish. In addition, cell growth was evaluated for differences in each culture condition. After three days of culture, cells reached confluence, so the cell number after two days was counted. As shown in Figure 6, there was no difference in cell proliferation rate and morphology owing to differences in culture methods. After two days of culture, there was no significant difference in cell number, so there was no device advantage for cell growth at this stage. At $1.4 \mu \mathrm{L} / \mathrm{min}$ set in the experiment, we assume that the pressure was hardly applied because the medium did not leak from the CD-Adapter even when the tube was removed from the CD-Adapter.

Overall, these results demonstrate that perfusion cultures using this novel system decreased cell stress and created improved culturing environment for cells compared with conventional manual medium change techniques. 
These results indicate that perfusion culture with the system reduced the stress on cells, suggesting that the disorderly medium replacement by the manual method may be the cause of stress.

A)

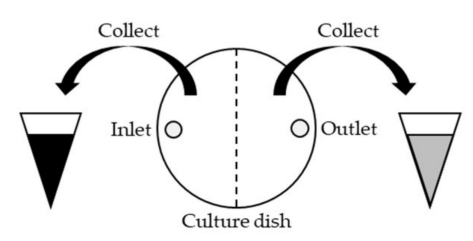

B)

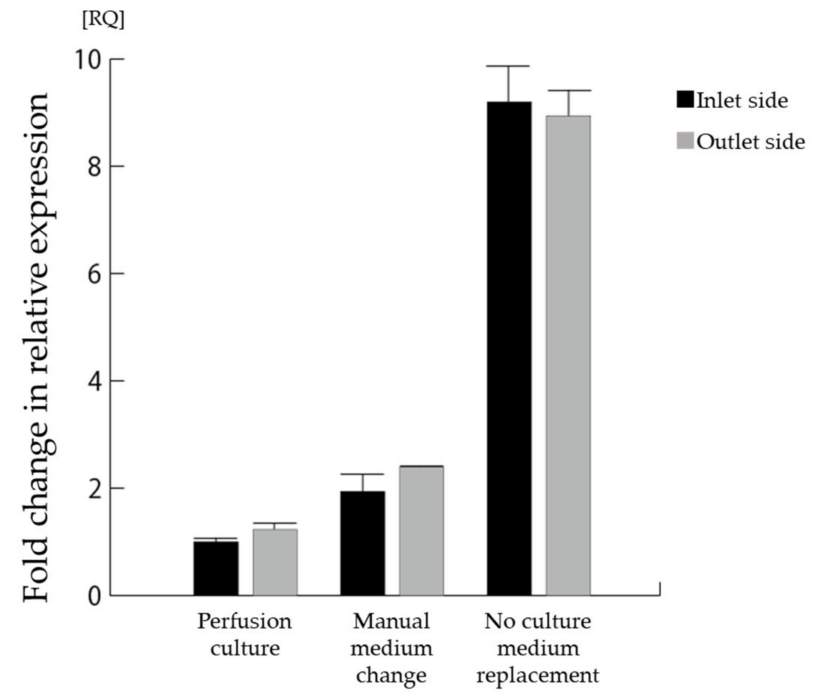

Figure 5. Gene expression analysis results. (A) Cell collection area on a culture dish. Cells cultured under each condition were collected from two halves of the culture dish: the inlet side and the outlet side. (B) Gene expression values of DDIT3 by 293T cells cultured for three days. The three experimental conditions are as follows: perfusion culturing with the system, culturing by manual medium change, and culturing without medium exchange. Each value was obtained by qRT-PCR. CD-Adapters were applied to all dishes to standardize the shape of the culture container.

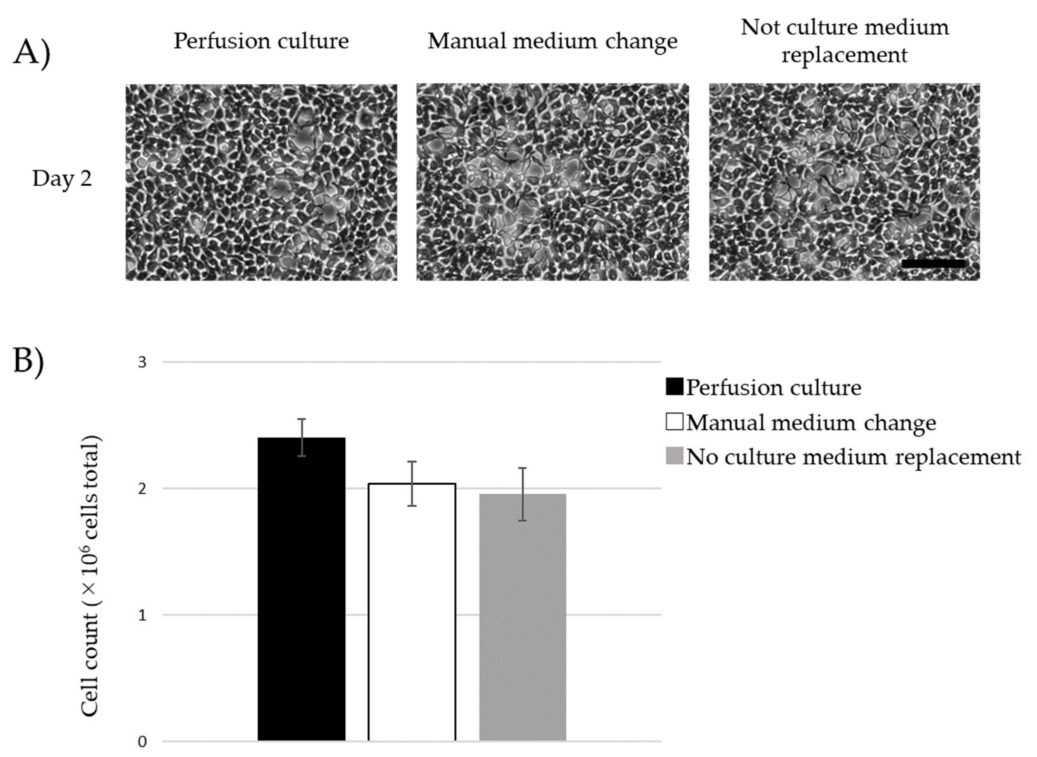

Figure 6. Microscopic image and cell growth rate of cultured 293T cells. (A) Results observed at $\times 40$ magnification. There was no difference in cell morphology, but the number of perfused cultured cells was larger than under other conditions. The bar is $10 \mu \mathrm{m}$. (B) The total number of cells was measured on the second days of culture, but there was no significant difference in each condition. 


\section{Conclusions}

In this study, we developed a novel perfusion culture system that is compatible with every $35 \mathrm{~mm}$ culture dish manufactured by companies. It was successfully confirmed that the holding jig that was inserted into the CD-Adapter prevented unintentional deformation of the cell culturing container. Additionally, the holding jig helps the CD-Adapter adapt to various $35 \mathrm{~mm}$ commercial culture dishes from different manufacturing companies, which vary from each other in size, by changing the size of the jig. By changing the spacer thickness, the amount of medium in the sealed culture space can be changed arbitrarily. Therefore, the system developed in this study may be compatible with various experimental conditions.

Additionally, we were able to verify the improvement of stress level in cells by applying the system for $293 \mathrm{~T}$ culturing. The results show that this system created a low-stress environment for the cells. The detailed mechanism of the relationship between the culture method and stress in the cells is still unknown and there is room for more research. Comprehensive evaluation including cell stress should be performed involving more types of cells, long-term culture cells, and iPS cells. Furthermore, when multiple pumps are used in combination, handling of the equipment surfaces is an issue owing to the increase in the number of tubes that needs to be resolved. In the future, we aim to create an optimal cell culture environment for basic research and regenerative medicine applications by this novel perfusion-culture system. If this device is applied to cells that require long-term culture, automation can be expected to reduce labor costs. In addition, as the closed culture environment can be created, it is not necessary to prepare a large-scale experimental facility, and the cost of equipment can be reduced. In addition to these, this device reduces the stress on cultured cells; thus, if used for the production of cell sheets in the field of regenerative medicine, it will be possible to mass-produce inexpensive and homogeneous cell sheets.

Author Contributions: Conceptualization, R.Y., K.M. and S.A.; formal analysis, R.Y. and K.M.; funding acquisition, A.O., S.A., K.K. and T.N.; investigation, R.Y.; methodology, R.Y. and K.M.; project administration, R.Y.; resources, Y.A. and T.K.; supervision, N.H. and T.N.; validation, R.Y.; visualization, R.Y.; writing-original draft preparation, R.Y.; writing-review and editing, K.M., S.A., N.H. and T.N. All authors have read and agreed to the published version of the manuscript.

Funding: The development of this perfusion system was supported in part by a Grant-in-Aid for Sample creation support project No. 408005 of the New Energy and Industrial Technology Development Organization (NEDO) of Japan; and the Adaptable and Seamless Technology Transfer Program through Target-driven R \& D (A-STEP) No. AS2815006U from the Japan Science and Technology Agency (JST). This work was also partially supported by Leading Initiative for Excellent Young Researchers (LEADER) in FY 2016 of the Ministry of Education, Culture, Sports, Science, and Technology, Japan.

Acknowledgments: The authors would like to thank H. Kotera and H. Shintaku (Institute of Physical and Chemical Research) for assistance with the fluid simulations.

Conflicts of Interest: Reiko Yasuda, Atsuhito Okonogi, and Yohei Anzai are employees of ICOMES LAB Co., Ltd., manufacturer of tube pump. Tadataka Kamiyama is director and Keiji Katano is a representative of ICOMES LAB Co., Ltd.

\section{References}

1. Unchern, S. Basic techniques in animal cell culture. In Proceedings of the Drug Delivery System Workshop, Bangkok, Thailand, 19-20 August 1999; pp. 1-30.

2. Ciraldo, F.E.; Boccardi, E.; Melli, V.; Westhauser, F.; Boccaccini, A.R. Tackling bioactive glass excessive in vitro bioreactivity: Preconditioning approaches for cell culture tests. Acta Biomater. 2018, 75, 3-10. [CrossRef] [PubMed]

3. Lee, H.J.; Diaz, M.F.; Price, K.M.; Ozuna, J.A.; Zhang, S.; Sevick-Muraca, E.M.; Hagan, J.P.; Wenzel, P.L. Fluid shear stress activates YAP1 to promote cancer cell motility. Nat. Commun. 2017, 8, 14122. [CrossRef] [PubMed]

4. Yoon, S.K.; Kim, S.H.; Lee, G.M. Effect of low culture temperature on specific productivity and transcription level of anti-4-1BB antibody in recombinant Chinese hamster ovary cells. Biotechnol. Prog. 2003, 19, 1383-1386. [CrossRef] [PubMed] 
5. Toh, Y.-C.; Voldman, J. Fluid shear stress primes mouse embryonic stem cells for differentiation in a self-renewing environment via heparan sulfate proteoglycans transduction. FASEB J. 2011, 25, 1208-1217. [CrossRef] [PubMed]

6. Ouyang, A.; Ng, R.; Yang, S.-T. Long-Term Culturing of Undifferentiated Embryonic Stem Cells in Conditioned Media and Three-Dimensional Fibrous Matrices without Extracellular Matrix Coating. Stem Cells 2007, 25, 447-454. [CrossRef] [PubMed]

7. Libertini, G. Phylogeny of aging and related phenoptotic phenomena. Biochemistry 2015, 80, 1529-1546. [CrossRef] [PubMed]

8. Sukho, P.; Cohen, A.; Hesselink, J.W.; Kirpensteijn, J.; Verseijden, F.; Bastiaansen-Jenniskens, Y.M. Adipose tissue-derived stem cell sheet application for tissue healing in vivo: A systematic review. Tissue Eng. Part B Rev. 2017, 24, 35-72. [CrossRef] [PubMed]

9. Moore, A.; Mercer, J.; Dutina, G.; Donahue, C.J.; Bauer, K.D.; Mather, J.P.; Etcheverry, T.; Ryll, T. Effects of temperature shift on cell cycle, apoptosis and nucleotide pools in $\mathrm{CHO}$ cell batch cultues. Cytotechnology 1997, 23, 47-54. [CrossRef] [PubMed]

10. Chow, J.M.; Russell, J.B. Effect of ionophores and $\mathrm{pH}$ on growth of Streptococcus bovis in batch and continuous culture. Appl. Environ. Microbiol. 1990, 56, 1588-1593. [PubMed]

11. Yu, H.; Meyvantsson, I.; Shkel, I.A.; Beebe, D.J. Diffusion dependent cell behavior in microenvironments. Lab Chip 2005, 5, 1089. [CrossRef] [PubMed]

12. Mogi, K.; Kanetaka, N.; Sugii, Y.; Hishida, K. Measurement of concentration distribution in endothelial surface layer using super resolution LIF technique. Trans. JSME 2015, 82, 15-00404.

13. Kondo, E.; Wada, K.-I.; Hosokawa, K.; Maeda, M. Microfluidic perfusion cell culture system confined in $35 \mathrm{~mm}$ culture dish for standard biological laboratories. J. Biosci. Bioeng. 2014, 118, 356-358. [CrossRef] [PubMed]

14. Huang, S.-B.; Chou, D.; Chang, Y.-H.; Li, K.-C.; Chiu, T.-K.; Ventikos, Y.; Wu, M.-H. Development of a pneumatically driven active cover lid for multi-well microplates for use in perfusion three-dimensional cell culture. Sci. Rep. 2015, 5, 18352. [CrossRef] [PubMed]

15. Saalfrank, D.; Konduri, A.K.; Latifi, S.; Habibey, R.; Golabchi, A.; Martiniuc, A.V.; Knoll, A.; Ingebrandt, S.; Blau, A. Incubator-independent cell-culture perfusion platform for continuous long-term microelectrode array electrophysiology and time-lapse imaging. R. Soc. Open Sci. 2015, 2, 150031. [CrossRef] [PubMed]

16. Yasuda, R.; Adachi, S.; Okonogi, A.; Anzai, Y.; Kamiyama, T.; Katano, K.; Hoshi, N.; Natsume, T.; Mogi, K. Fabrication of a Novel Culture Dish Adapter with a Small Recess Structure for Flow Control in a Closed Environment. Appl. Sci. 2019, 9, 269. [CrossRef]

17. Pakos-Zebrucka, K.; Koryga, I.; Mnich, K.; Ljujic, M.; Samali, A.; Gorman, A.M. The integrated stress response. EMBO Rep. 2016, 17, 1374-1395. [CrossRef] [PubMed]

18. Vihervaara, A.; Duarte, F.M.; Lis, J.T. Molecular mechanisms driving transcriptional stress responses. Nat. Rev. Genet. 2018, 19, 385-397. [CrossRef] [PubMed]

(C) 2019 by the authors. Licensee MDPI, Basel, Switzerland. This article is an open access article distributed under the terms and conditions of the Creative Commons Attribution (CC BY) license (http://creativecommons.org/licenses/by/4.0/). 